\title{
From Event Structure to Scale Structure: Degree Modification in Deverbal Adjectives
}

\author{
Christopher Kennedy and Louise McNally \\ Northwestern University and Universitat Pompeu Fabra
}

\section{Introduction}

An issue of interest to work in areas ranging from lexical semantics to natural language processing (especially automatic text generation) to language pedagogy is the semantics of degree modification by expressions like very, well, and much. Focusing on the case of deverbal gradable adjectives (such as acquainted), we show that the selective behavior of different degree modifiers provides an important probe on the semantic typology of adjectives. Specifically, we demonstrate that the distribution and interpretation of degree modifiers is sensitive both to the scalar structure of the adjectives they modify and to the nature of the "standard values" according to which sentences involving adjectival predications are judged to be true. We further show that the scalar structure of deverbal gradable adjectives is determined by the aspectual properties of the source verbs, making it possible to accurately predict both which degree modifiers will be acceptable with which participles, and how they will be interpreted.

\section{Degree Modification in Deverbal Gradable Adjectives}

The empirical starting point of this paper is three puzzles involving the acceptability of degree modification of deverbal gradable adjectives by well, very, and much. First, why do the participles in (1) accept degree modification by well but not very?

(1) a. Martin Beck is well/??very acquainted with the facts of the case.

b. The facts are well/??very understood.

c. The concert was well/??very publicized.

d. The abuse of public funds was well/??very documented.

The acceptability judgments in (1) are mirrored by corpus data: as shown in (2), there is a clear statistical tendency for such participles to appear with well rather than very.

(2) a. educated: 3 very (2 attributive, both from same text) vs. 78 well in the British National Corpus (BNC)

b. defined: 2 very (both attributive) vs. 146 well in the BNC

c. protected: 2 very vs. 62 well in the $\mathrm{BNC}$ 
Second, what governs the distribution of the degree modifier much? As shown by the examples in (3), the distribution of much is largely complementary to that of both degree well and very:

(3). a. a much/??well/??very desired position

b. a much/??well/??very needed rest

c. a much/??well/??very praised film

d. a much/??well/??very talked about novel

(4) a. ??much/very tall/expensive/happy

b. ??much/well acquainted/understood/paid

As with well, one also finds numerous examples from the BNC in which a participle shows a strong statistical tendency to favor much over other possible degree modifiers:

(5) a. needed: 2 well vs. 211 much

b. appreciated: 12 well vs. 134 much

c. prized: 1 very vs. 16 much

The final question concerns the interpretation of well-modification. While well has a degree reading in the sentences in (1) (i.e., (1a) means that Beck is acquainted with the facts to a fairly high degree), in the examples in (6), it does not, but instead has a "quality" or "manner" interpretation. (6c), for example, means that the house was built in a high quality way, not that its construction was high on a scale of completion.

(6) a. The suit was well cut.

b. The book was well written.

c. The house was well built.

An initial response to these facts, in particular to the unacceptability of modification by very in the examples in (1) and (3), would be to claim that these participles simply are not adjectives, or at least are not gradable adjectives. As shown by the examples in (7), neither true verbal participles nor nongradable adjectives permit modification by very.

(7) a. ?? The president was very impeached by the House of Representatives.

b. ?? Richard Nixon, a very former president, resigned before he was impeached.

. This response cannot be correct, however. First, the facts in (8) show that the participles that disallow modification by very allow un-prefixation, a property of adjectives, not verbs. 
(8) a. Beck is unacquainted with the facts of the case.

b. The singer's unpublicized appearance caused a commotion at the restaurant.

c. These claims are undocumented, and therefore not admissible in court.

d. uneducated, undefined, unprotected

e. unneeded, undesired, unpraised, unappreciated

Second, they can appear as complements to copular verbs such as seem, remain or become, yet another adjectival property.

(9) a. Beck seemed acquainted with the facts of the case.

b. The phenomenon remains poorly understood.

c. The scandal became publicized after a leak to the press.

d. The case remained documented on file.

Finally, these participles appear in comparative constructions, a property that is true only of gradable adjectives. This is illustrated by the corpus data in (10).

(10) a. But as I became more acquainted with this set and stopped rushing from impossible passage to impossible passage, hoping against hope that at some point he would lose his balance and tumble like a secondrate trapeze artist off his swing, I was unwittingly dragged in to a more sinister, melancholic side to his playing. [CD Review, 1992. (BNC)]

b. The causes of weakness in adhesion are rather less understood at present than they are in cohesion but no doubt they are rather similar in character. [J. Gordon, The New Science of Strong Materials. 1991. (BNC)]

c. This was certainly more dramatic than the more publicized event that finished off the dinosaurs. [Antony Milne, The Fate of the Dinosaurs: New Perspectives in Evolution. 1971. (BNC)]

d. He was more talked about than if he had been open and obvious. [Jean Bow, Jane's Journey, 1991. (BNC)]

e. $\quad$...virginity was more prized, promiscuity was frowned upon. [W.F.R. Stewart, Sexual Aspects of Social Work, 1979. (BNC)]

We therefore conclude that the facts in (1)-(5) cannot be explained in terms of category mismatch: the deverbal expressions are gradable adjectives (see Borer 1998:92-3, for the same conclusion). In the rest of the paper, we will show that : the distinctions between very, well, and much are moreover not due to idiosyncratic properties of certain deverbal adjectives, but rather reflect deeper aspects of the semantics of degree modification, the scalar structure of gradable adjectives, and the 
relation between scale structure and the aspectual structure of verbs. In particular, we provide evidence for the following claims.

First, the three modifiers are each sensitive to different aspects of the scalar properties of the adjectives they modify. In the case of very and much, this is observed in the requirements they impose on the nature of their argument's "standard value", the degree with respect to which predications involving gradable adjectives are judged to be true or false, whose properties we show to be determined by the base adjective's scale structure. Very and much are both true degree modifiers which have the effect of "boosting" the standard associated with (a particular use of) a gradable adjective, but very selects for an adjective that has a context-sensitive standard, while much requires its argument to have a context-insensitive standard. Well, in contrast, is a conventional adverbial modifier that is more directly sensitive to the scale structure of its adjectival argument. It selects adjectives that have what we call a (totally) closed scale (a scale with a maximal and minimal value) and returns an adjective with an open scale (a scale without maximal/minimal values).

Second, we show that the class of deverbal adjectives with totally closed scales corresponds to the class of verbs that introduce incremental themes (Dowty 1991), and we argue that this connection can be explained in terms of the homomorphic relation between the progression of the event and (some property of) the incremental theme argument (see Krifka 1989, 1992, Ramchand 1997).

Finally, we demonstrate that the degree modifier reading of well is blocked when the standard for the participle it modifies corresponds to an upper endpoint of a scale, and we show that the orientation of the standard (upper or lower endpoint) can be predicted as a function of the semantic role that the target of predication has in the verbal form.

\section{Scale Structure and Standard Values}

\subsection{Standards of Comparison and Context Dependence}

As is well known, the interpretation of gradable adjectives like tall or inexpensive is highly context dependent: what "counts as" e.g. tall or inexpensive varies from context to context. One way to account for this variation is to characterize the meaning of a gradable adjective in terms of a contextually defined standard of comparison (see e.g. Sapir 1944, McConnell-Ginet 1973, Kamp 1975, Klein 1980, 1991, Bierwisch 1989, Ludlow 1989, Kennedy 1999 and others). On this view, sentences such as those in (11) are assigned truth conditions in (12).

(11) a. Michael Jordan is tall.

b. The Mars Pathfinder mission was inexpensive.

(12) a. Michael's height is at least as great as a standard of tallness (for basketball players). 
b. The cost of the Mars Pathfinder mission was at least as great as a standard of inexpensiveness (for missions to outer space).

This idea can be implemented by adopting a model in which the semantic analysis of gradable adjectives is stated in terms of abstract representations of measurements, or "scales", which are formalized as totally ordered sets of points, or "degrees" (see Klein 1991 for an overview). In this approach, the truth conditions of e.g. (11b) are as in (13), where inexpensive is a function from objects to degrees and $d_{s \text { (inexpensive) }}$ is a free variable over degrees that identifies a standard for the adjective (see Kennedy 1999 for a fully explicit compositional semantics for adjectival predicates along these lines; see also Bartsch and Vennemann 1973).

$$
\text { inexpensive }(m) \succeq d_{\text {s(inexpensive })}
$$

Since the value of the standard variable is fixed by the context, the truth of $(11 \mathrm{~b})$ may vary. It may be true in a conversation about the space program, for example, but false in a discussion about things with the name "Pathfinder" (which might include compasses, bicycles, and sport utility vehicles in addition to missions to Mars).

Not all gradable adjectives show the same sort of context sensitivity, however. The standard values for the adjectives in (14) appear to be fixed.
a. The baby is awake.
b. The cookie jar is empty.
c. The line is straight.

Under normal usage, (14a) does not mean that the degree to which the baby is awake surpasses some standard (for babies), but rather simply means that the baby has achieved some minimal level of "awakeness". Similarly, (14b) means that the cookie jar is completely empty, not that its contents fall below some standard of emptiness ((14c) is similar). Note that the context-independence of these adjectives does not indicate that they are not gradable: as shown by (15), they are perfectly felicitous in comparatives.

(15) a. The baby is more awake now than it was a few minutes ago.

b. The cookie jar is emptier than it was this morning.

c. The red line is straighter than the blue one.

What is responsible for this difference in context-sensitivity?

\subsection{Different Scales, Different Standards}

Intuitively, the difference between adjectives like inexpensive, tall, interesting, etc. and those in (14) is that the latter are conventionally associated with scales that allow mapping to an endpoint - a maximal or minimal degree - while the former are associated with open scales - scales without endpoints. Moreover, there is empirical 
evidence for this distinction. As illustrated by the contrasts in (16) and (17), adjectives such as empty, full, and awake differ from adjectives with context-insensitive standards with respect to the acceptability of modification by "proportional modifiers" like completely, partially, and half. ${ }^{1}$

(16) a. completely empty/full/awake

b. partially empty/full/awake

c. half empty/full/awake

a. ?? completely tall/short/interesting/inexpensive

b. ?? partially tall/short/long/interesting/inexpensive

c. ?? half tall/short/long/interesting/inexpensive

These facts can be explained as follows (cf. Lehrer 1985, Hay 1998). First, making more concrete the hypothesized difference in scale structure, assume that the adjectives in (16) map objects onto totally closed scales (scales that include two endpoints), while those in (17), map objects totally open scales (scales that exclude endpoints). Second, assume that the compositional semantics of proportional modifiers requires reference to two endpoints in order to compute the properties they describe. On this view, the examples in (17) are anomalous because open scale adjectives do not introduce the necessary endpoints.

With these distinctions in scale structure in mind, we can make the following generalization about the context sensitivity of the standard value: adjectives associated with open scales have context-sensitive standards; adjectives with closed scales have context-insensitive standards. More precisely, the standard values for the latter adjectives default to an endpoint of the scale (the lower point for e.g. awake and the upper point for e.g. full and straight; we return to a discussion of the orientation of the standard in section 6). To distinguish between these two types of adjectives, we introduce the terminology in (18).

(18) a. An adjective has a trivial standard iff its standard defaults to an endpoint of the scale.

b. An adjective has a nontrivial standard iff its standard is context dependent.

Entailment patterns provide a test for determining whether a particular adjective has a trivial or nontrivial standard. The standard semantic analysis of (noncomparative) adjectival predications has the effect that expressions of the form $x$ is $\alpha$ and $x$ is not $\alpha$ have the truth conditions in (19) (see the discussion of (13) above).

$\begin{aligned} \text { (19) } \quad \text { a. } & \llbracket x \text { is } \alpha \rrbracket=1 \text { iff } \alpha(x) \succeq d_{s(\alpha)} \\ \text { b. } & \llbracket x \text { is not } \alpha \rrbracket=1 \text { iff } \alpha(x) \prec d_{s(\alpha)}\end{aligned}$ 
It follows that for an adjective $\alpha$ with a trivial standard, if this standard corresponds to the lower end of the scale, a statement of the form $x$ is not $\alpha$ should entail that $x$ has no amount of " $\alpha$-ness" at all (see (20a), where "\#" denotes contradiction). ${ }^{2}$ If $\alpha$ 's trivial standard corresponds to the upper end of the scale, however, then $x$ is $\alpha$ should entail that $x$ has a maximal amount of " $\alpha$-ness" (see (20b) and note 1). Neither of these entailments should hold, however, if $\alpha$ has a nontrivial standard (see (21)).

(20) a. \# My hands are not wet, but there is a little bit of water on them.

b. \#The candle is straight, but you could make it straighter.

(21) a. Sam is not tall, but his height is normal for his age.

b. That film is interesting, but it could be more interesting.

One consequence of the definitions in (18) is that if an adjective has a trivial standard, then it must have a scale which is closed on at least one end. In contrast, our research so far indicates that the implication in the other direction is simply a very strong statistical tendency: adjectives with closed or partially closed scales have trivial standards as a default, but this default can, in certain circumstances, be overridden. ${ }^{3}$ The broader conclusion to be drawn from the discussion in this section is that there is a direct correlation between scale structure and one of the most fundamental semantic properties of gradable adjectives: the context dependency of their standard values. As we will see in the next section, the relation between scale structure and standards also supports an explanation of the distribution of the degree modifiers very, much, and well.

\section{The Semantics of Degree Modifiers}

\subsection{Very and Well}

Roughly speaking, the difference between e.g. expensive and very expensive is that the latter denotes a property whose meaning is just like the former, except that the standard value is "boosted" by some amount. This is most clearly illustrated by pairs like the one in (22), which shows that the "standard boosting effect" of very (in terms of absolute increase) depends on how high the initial standard value is.

(22) a. The international space station is very expensive. (for space projects; large increase in the standard)

b. The coffee at the airport is very expensive. (for coffee; smaller increase in the standard)

The connection between very and the standard value is not unrestricted, however: in normal usage, adjectives associated with trivial standards reject modification by very: 
(23) a. ?? They were very able to solve their own problems.

b. ?? The baby is very awake. ( $\neq$ wide awake)

To account for these facts, we propose that very presupposes that the adjective it modifies is associated with a nontrivial standard. This hypothesis is implemented in the semantic analysis in (24), in which $G$ is a function from objects to degrees (a gradable adjective meaning), $d_{s}$ is a standard, and LARGE is a context-dependent function that identifies the amount by which the standard value should be increased (see Hellan 1981, von Stechow 1984b; and Klein 1991 for formal approaches to degree addition).

$$
\begin{aligned}
& \llbracket v e r y \rrbracket=\left\{\left\langle G,\left\langle d_{s(G)}, x\right\rangle\right\rangle \mid \exists d\left[G(x) \succeq d_{s(G)}+d \wedge \operatorname{LARGE}(d)\right]\right\} \\
& \text { CONDITION: } d_{s(G)} \text { is nontrivial }
\end{aligned}
$$

In contrast to very, well combines felicitously with adjectives that have totally closed scales, but not with adjectives that have open scales: ${ }^{4}$

(25) a. We are well aware of the difficulties.

b. They are well able to solve their own problems.

(26) a. ?? The international space station is well expensive.

b. ?? Michael Jordan is well tall.

Note also that the output of well modification, unlike very modification, supports a full range of further degree modification, including participation in comparative constructions:

(27) a. They remained very/quite/only too/hardly well aware of the difficulties that might arise from their analysis.

b. Sam is more well able to cope with the situation than is his brother.

(28) a. ?? They became quite/only too/hardly very happy at the news.

b. ?? Michael Jordan is less very tall than Shaquille.

We account for these facts by analyzing well not as a degree modifier but rather as a function from (gradable) adjective meanings to adjective meanings, such that the input is associated with a totally closed scale and the output is associated with a totally open scale, as stated in (29).

(29) $\llbracket w e l l \rrbracket=\left\{\left\langle G, G^{\prime}\right\rangle \mid G\right.$ is a function from objects to closed scales and $G^{\prime}$ is a function from objects to open scales $\}$

. Given the correlation between scale structure and standard values observed in the previous section, the result of this proposal is that adjectival expressions of the form well $(G)$ should have nontrivial standards (which, moreover, the semantics of 
well apparently presupposes are relatively high), and they should therefore permit modification by very. The data discussed above show that this is indeed the case. ${ }^{5}$

The semantic analyses of very and well outlined lead to an answer to the first of the three questions we asked at the beginning of this paper. Recall that the data in (1) showed that modification of certain deverbal gradable adjectives by very is infelicitous, while modification by well is possible. The examples in (30) extend this descriptive generalization, demonstrating that modification of the well $A$ complex by very is also possible.

(30) a. Martin Beck is very well acquainted with the facts of the case.

b. The facts are very well understood.

c. The concert was very well publicized.

d. The abuse of public funds was very well documented.

According to the semantic analyses of very and well proposed above, the facts in (1) and (30) follow if these participles are associated with trivial standards and totally closed scales. That such participles have totally closed scales is demonstrated by the acceptability of proportional modification:

(31) a. Beck is completely/fully/partially acquainted with the facts of the case.

b. Language change is completely/fully/partially understood.

c. The concert was completely/fully/partially publicized in the mass media.

d. Those war crimes are completely/fully/partially documented.

That these participles have trivial standards is demonstrated by their entailment patterns (see the discussion in section 3):

(32) a. \# Beck isn't acquainted with the facts facts of the case, though I did show him the coroner's report.

b. \# The importance of the Dolly experiments is not understood, though we know that the data suggests that it might be possible to clone humans.

c. \# The concert was not publicized, but there were a few posters in the metro announcing it.

d. \# The details of the murder were not documented, though the police reports contain a record of a weapon at the scene of the crime.

The answer to the first question raised in section 1, then, is that gradable adjectives like acquainted, understood, publicized and so forth accept modification by well but not very because (1) they are associated with totally closed scales, and (2) they have trivial standards. 


\subsection{Much}

The distribution of the output of much modification strongly suggests that much is a degree modifier similar to very. For example, like the output of very modification, and unlike the output of well modification, expressions of the form much A cannot be further modified and do not participate in comparative constructions:

(33) a. ?? Your help is quite/only too/hardly much needed.

b. ?? My vacation is more much needed than yours.

However, there is an important difference between the two, and a corresponding similarity between much and well: much only modifies adjectives with trivial standards. This proposal is made explicit in (34).

$$
\begin{aligned}
& \llbracket m u c h \rrbracket=\left\{\left\langle G,\left\langle d_{s(G)}, x\right\rangle\right\rangle \mid \exists d\left[G(x) \succeq d_{s(G)}+d \wedge \operatorname{LARGE}(d)\right]\right\} \\
& \text { CONDITION: } d_{s(G)} \text { is trivial }
\end{aligned}
$$

This analysis makes two important predictions. First, if much is a standardboosting expression, we predict that it cannot felicitously modify an adjective whose standard corresponds to an upper endpoint of a scale, since upper endpoint standards are maximal, and so cannot be boosted; it may only modify those with lower endpoint standards. The behavior of the participles that accept modification by much with respect to the contradiction test indicates not only that their standards are trivial, but also that they are systematically lower endpoints:

(35) a. \# The war was not desired, but certain parties hoped that a conflict would break out.

b. \# Your financial support is not needed, but we could use a small contribution from you.

c. \# The film was not praised, but one critic said good things about it.

d. \# The problem was not talked about, though Frank mentioned it to his mother.

Second, if much differs from very only in the nature of the standard value, nothing should require the modified adjective's scale to be closed on the upper end. This leads to the prediction that much should be compatible with adjectives that have simply partially closed scales. This prediction is also borne out: the participles that were shown in (3) and (5) to prefer modification by much are unacceptable with proportional modifiers, which shows that they do not have totally closed scales:
a. ?? a completely/fully/partially needed rest
b. ?? a completely/fully/partially desired result
c. ?? a completely/fully/partially praised film
d. ?? a completely/fully/partially talked about novel 
We therefore have an answer to our second question: the distribution of much is complementary to that of very because the two are sensitive to different kinds of standards. ${ }^{6}$ It will be at least partially complementary to that of well, since, unlike the latter, it is compatible with scales that are closed only on one end. It remains to be investigated to what extent much is compatible with adjectives whose scales are closed on both ends. As stated, the semantics for much does not prevent it from being compatible with totally closed scales as well, and thus allows for overlap between the distribution of much and well. However, facts such as those in (4) may ultimately be evidence that much selects not just for a trivial standard, but for a scale which is only partially (and crucially not totally) closed.

\subsection{Summary}

The interaction of scale structure, standard values, and the distribution of degree modifiers is summarized in (37).

$$
\begin{aligned}
& \text { a. TOTALLY OPEN SCALE - NONTRIVIAL STANDARD }-\sqrt{ } \text { very - } \\
& \text { ??well }- \text { ??much } \\
& \text { b. TOTALLY CLOSED SCALE }- \text { TRIVIAL STANDARD }- \text { ??very }-\sqrt{ } \text { well } \\
& \quad \text { ??much } \\
& \text { PARTIALLY CLOSED SCALE }- \text { TRIVIAL STANDARD }- \text { ??very - } \\
& \text { ??well }-\sqrt{ } \text { much }
\end{aligned}
$$

If these generalizations are valid, as the facts discussed here strongly indicate, then they provide an important tool for identifying the lexical semantic properties of gradable adjectives (derived or otherwise) on the basis of collocational patterns. More generally, the range of facts involving very, well, and much indicate that three of the four logically possible (linear) scale types are used in natural language: totally open scales, totally closed scales, scales which are closed only on the bottom end. An additional question for future research is whether there exist adjectives whose scales are closed only on the top end, and if so, whether there any degree modifiers which are sensitive to them.

\section{From Event Structure to Scale Structure}

The previous section argued that the distribution of degree modifiers is directly or indirectly determined by aspects of scale structure: whether a scale is open or closed, and how scale structure interacts with the trivial/nontrivial standard distinction. Within the context of the empirical focus of this paper-the case of deverbal adjectives-this analysis immediately raises a new question: why do particular deverbal adjectives have the scalar structures that they have? In fact, further observa-

$:$ tion reveals striking correlations between a participle's scalar structure and certain 
characteristics of the situation described by the verb from which that participle derives. We thus hypothesize that we can, to a significant degree, predict the scalar structure of a participial adjective.

First, the data that we have observed indicate that the class of deverbal adjectives that have totally closed scales corresponds very closely to the class of verbs that introduce incremental themes. As pointed out by $\operatorname{Krifka}(1989,1992)$ (see also Dowty 1991, Tenny 1995, Jackendoff 1996, and Ramchand 1997), what is unique about this class of verbs is that it is possible to establish a homomorphic relationship between the events they denote and (some measurable property of) their incremental theme arguments (see Hay, Kennedy, and Levin this volume). ${ }^{7}$ We claim that it is precisely this homomorphism that is responsible for the scalar properties of the derived adjectives, because it provides a template for building a closed scale, specifically a scale with a lower endpoint that corresponds to the minimal (sub)event involving (a minimal part of) the incremental theme or the relevant measurable property, and an upper endpoint that corresponds to the maximal event involving (all of) the incremental theme/property. (That the aspectual properties of verbs affect the semantics of derived adjectives is also claimed by Yumoto (1991) to explain the distribution of adjectival passives.)

For example, consider loaded, as in the truck is loaded with hay. Let us assume, generalizing Dowty's (1991) analysis of spray/load verbs, that the truck is the incremental theme in the situation described. We can define a mapping between the progress of the event of loading and a property of the truck-namely, the volume of the material that it holds; the degree to which the truck can be said to be loaded corresponds to the degree to which it has progressed through a loading event. Since we can define a beginning point and endpoint for this event (corresponding to when the truck is empty and full, respectively), we can identify a lower bound and upper bound for the scale of "loadedness" of the truck.

Since, for reasons of space, we cannot exhaustively demonstrate the generality of the correlation between event structure and scale structure, we will simply consider some representative adjectival participles from various verb classes. ${ }^{8}$ First, let us consider the implication that, if a participial adjective has a closed scale, it is derived from a verb that has an incremental theme. As discussed in section 3.2, closed-scale adjectives are identifiable by compatibility with proportional modifiers like completely and partially. If this implication is correct, we expect it to be impossible to say completely/fully/partially $A$, where $A$ is a participle derived from a verb lacking an incremental theme. And indeed, the examples we have found, such as those illustrated in (38), systematically bear out this prediction. ${ }^{9}$ The participles in (38) include states ((38a)-(38c)), activities ((38d)-(38f)), semelfactives ((38g)), and change of state predicates in which the theme is affected wholistically and whose result state is associated with an open scale (such as worry) $((38 \mathrm{~h}))$.
a. ?? a completely hated/loved/envied/admired neighbor
b. ?? a fully needed/wanted rest
c. ?? a partially regretted action 
d. ?? a completely looked for/expected reaction

e. ?? a fully driven/pushed car

f. ?? a completely watched suspect

g. ?? a partially $\mathrm{kissed} / \mathrm{met} /$ punched young man

h. ?? a completely worried/pleased/surprised mother

Now consider the implication that, if a participial adjective is derived from a verb that has an incremental theme, it has a closed scale. As the examples in (39) show, this implication also holds quite generally.
a. a partially eaten meal
b. a fully written novel
c. completely loaded hay
d. a completely paid bill
e. fully straightened teeth
f. a partially heard response
g. a partially anticipated reply
h. a fully understood problem

Notice that while some of these participles, such as eaten, correspond to prototypical incremental theme verbs, others, such as straightened or heard, do not. The members of this latter class of verbs do, however, have arguments that share an important property with canonical incremental themes: they possess properties that can be homomorphically related to the structure of the corresponding event (e.g., the straightness of the teeth; the amount of the response that has been heard, and so forth; see Hay, Kennedy, and Levin this volume). Given our remarks above, then, it is not surprising that these verbs pattern with the more prototypical incremental theme verbs with respect to the semantics of their adjectival forms. ${ }^{10}$

The robustness of our generalizations could be challenged by the fact that certain participles derived from incremental theme verbs do cooccur with very, as noted in (2) and as seen in examples such as a well/very balanced diet. However, recall that the distribution of well and very depends on the type of standard associated with the adjective and not on the nature of the scale. It simply happens that closed scales have a strong tendency to be associated with trivial standards. Our analysis thus allows for the possibility that some participles may occur with both modifiers, but makes the prediction that the choice of modifier should indicate what type of standard the speaker is using to evaluate the adjectival property. For example, if we talk about a very balanced diet, we are committed to the existence of a contextually determined standard of "balancedness" which does not entail (or even necessarily have anything to do with) the diet's having participated in a minimal event of being balanced. When we talk about a balanced tire, however, there must be a balancing event involving the described object, thus the anomaly of ?? a very balanced tire. 
The relationship between event structure and scale structure is also manifest when we consider those participial adjectives with partially closed scales, such as needed: these adjectives are routinely derived from atelic verbs, whether state- or activity-denoting. This is exactly what we expect. The lower endpoint of a partially closed scale corresponds to a minimal (sub)event or state which supports the truthful application of the adjectival property to its argument. For instance, in order to qualify as needed, an entity must stand in some minimal need relation to someone or something. However, since atelic verbs describe situations with no natural endpoint, there is no clear maximal event or state which could correspond to an upper endpoint of the corresponding adjectival scale. The scale is thus open on the upper end.

\section{The Orientation of the Trivial Standard and the Interpretation of well}

Now that we have addressed the question of why participial adjectives have the types of scales they do, we are left with one remaining theoretical question: whether we can predict the orientation of their standard values. This question turns out to be related to the third of the empirical puzzles presented at the beginning of the paper: why do some uses of well, such as (40a), have a degree reading, while others, such as $(40 \mathrm{~b})$, have a manner or quality reading instead?

(40) a. Beck is well acquainted with the facts.

b. The house was well built.

Our research shows that the answer to this question has to do with the "orientation" of the trivial standard. Specifically, when the trivial standard corresponds to the lower endpoint of a scale, a degree reading of well is available, but when the standard is the upper endpoint, this reading disappears. To see that this is so, observe that the entailment tests for upper vs. lower endpoint standards indicate that $a c$ quainted has a lower endpoint standard, while built has an upper endpoint standard:

a. \# Beck isn't acquainted with the facts facts of the case, though I did show him the coroner's report.

b. Beck is acquainted with the facts, though he is still missing some of the details.

c. The house isn't built yet, though the foundation has been laid.

d. \# The house is built, but it still needs a roof.

While we cannot pursue in detail here the question of why this correlation should exist, we can at least make some preliminary comments. Intuitively, degree modification by well induces a partitioning on the set of things which have an adjectival property $\alpha$, dividing them into those things which are $\alpha$ and fall above the standard established by the addition of well, and those which are $\alpha$ to a lesser degree, and so do not meet this standard. Now, if a particular adjective $\alpha$ can be 
truthfully predicated only of objects that are mapped to the maximum endpoint on a scale - a property of adjectives with upper-endpoint trivial standards - then it is impossible for well to induce the type of partition described above, since the relevant distinction cannot be made among things which, in order to qualify as having $\alpha$ at all, must be maximally $\alpha$. It follows that modification by well should be unacceptable on the degree reading, although there would be no reason in principle for such modification not to take on a manner or quality reading.

The sensitivity of the interpretation of well to the orientation of the standard leads us to ask if it is possible to predict whether an adjective's (trivial) standard corresponds to an upper or lower endpoint based on other aspects of its semantics. It turns out that it is. Specifically, the orientation of the standard depends on the role of the participial adjective's argument in the event associated with the corresponding verb: (participial) adjectives (with trivial standards) whose arguments satisfy Krifka's (1989) Mapping to Objects (see note 7), such as cut and written, have upper endpoints as standards, while those whose arguments do not, such as acquainted and documented, have lower endpoints as standards. This is most clearly illustrated by the pairs in (42)-(43), in which the argument of the adjective in the (a) sentence does not satisfy Mapping to Objects, while the argument in the (b) sentence does.

(42) a. Jones is well prepared for her talk. (degree reading possible)

b. Jones' talk is well prepared. (no degree reading)

a. a well-loaded truck (degree reading possible)

b. well loaded hay (no degree reading)

The explanation for this distinction can be traced to aspects of the verbal predications. Consider first the case of the argument satisfying Mapping to Objects. Because it cannot be asserted that the eventuality corresponding to the participle is completed until the argument has been totally affected (in the relevant way), it follows that an adjectival participle truthfully applies to such an argument only if that argument possesses a maximal amount of the relevant (deverbal) property. The result is an upper endpoint standard.

The situation is different in the case of other types of arguments. Since the completion of the eventuality corresponding to the participle does not depend on affecting all of the relevant argument (or affecting that argument in its entirety), it may be asserted that the eventuality is completed even when that argument has been minimally affected. As a result, the adjectival participle may be truthfully applied to such an argument as long as the argument possesses a minimal degree of the relevant property. This derives a lower endpoint standard.

\section{Conclusion}

The work reported here illustrates some of the benefits to be gained from investigating even a very small lexical semantic phenomenon. Focusing on the distribution of 
the degree modifiers very, well, and much in the context of deverbal gradable adjectives, we have gained insight into more general aspects of scalar structure to which natural language, and gradable adjectives in particular, are sensitive. In particular, our study has demonstrated that there is a strong correlation between an adjective's scalar structure (whether it is associated with a totally or partially closed vs. a totally open scale), the context sensitivity of its standard value (whether it has a trivial or nontrivial standard), and the acceptability and interpretation of degree modification by very, well, and much. These observations provide a strong basis both for making predictions about the lexical semantic properties of gradable adjectives (derived or otherwise) on the basis of collocational patterns, and for determining which degree modifiers will be acceptable with which participles. Finally, we have provided new insight into the relationship between the aspectual structure of verbs and the scalar structure of adjectives.

\section{Endnotes}

* We are grateful to Beth Levin and audiences at the University of California, Santa Cruz, University of Southern California, Northwestern University, Universitat Pompeu Fabra, and the Universitat Autònoma Barcelona for very helpful comments on the material discussed in this paper.

1. It should be observed that maximality modifiers like completely and totally have both an endpoint-oriented use and a use that is roughly synonymous with very; these two uses are distinguished by their entailments. An endpoint-oriented use entails that the end of a scale has been reached; thus, the sentence 'The line is completely/totally straight, though you can make it straighter' is a contradiction. A non-endpoint-oriented use carries no such entailment, thus the contingency of ' $I$ ' $m$ completely/totally uninterested in finances, and Bob is even less interested than me'. 2. The only way the ordering relation in (19b) could fail to hold for a lower-endpoint standard is if the result of applying $\alpha$ to $x$ were no value at all, i.e. the "empty degree". This result can be straightforwardly derived in a model in which degrees are formalized as intervals (sets of points) on a scale, as in Seuren 1978, von Stechow 1984b, and Kennedy 1999. In this type of approach, the empty degree is simply the empty set, which satisfies the ordering relation in (19b) for a lower-endpint $d_{s(\alpha)}$.

3. There is a very plausible functional explanation for this: since the endpoint of the scale provides a natural and fixed reference point to use as a standard, closed scale adjectives are conventionally associated with trivial standards. Such a strategy is unavailable to open scale adjectives, however; therefore their standards must be context dependent.

4. Not all nonderived adjectives with totally closed scales permit modification by well, but this is possibly due to the independent morphosyntactic preference for well to modify participles. See Bolinger 1972:38ff.

5. In contrast, according to the analysis of very in (24) and the analysis of much that we will present below in (34), the constituent very/much adj is of the wrong semantic type to combine with a degree modifier, since it denotes a set of individuals 
and not a simple measure function, like plain adjectives.

6. The hypothesis that much is sensitive to trivial standards may also explain the fact that it appears so commonly with comparatives (much happier, much shorter, etc.), which differ most obviously from non-comparatives in that their standards are not context-dependent, but rather are named (by the comparative clause).

7. Krifka (1989:92) captures this homomorphism formally in terms of his notion of "Mapping to Objects", defined as a characteristic of thematic roles $R$ as follows:

$$
\forall R\left[\mathbf{M A P}-\mathbf{O}(R) \leftrightarrow \forall e \forall e^{\prime} \forall x\left[R(e, x) \wedge e^{\prime} \subseteq_{E} e \rightarrow \exists x^{\prime}\left[x^{\prime} \subseteq_{O} x \wedge R\left(e^{\prime}, x^{\prime}\right)\right]\right]\right]
$$

In prose MAP-O guarantees that all subevents $e^{\prime}$ of a given event $e$ with participant $x$ in role $R$ will involve a part $x^{\prime}$ of $x$.

8. Note that we crucially consider only those participles which are demonstrably adjectives according to the tests used in section 2. See e.g. Levin and Rappaport 1986 on the question of which participles can be adjectival. Moreover, we will not explicitly demonstrate that these and the remaining participles discussed in this paper are adjectives. We simply point out that the majority of them accept unprefixation, and those which do not (like hated) occur readily as the complement to predicates like seem.

9. The only exception we have found to this prediction is known, which admits modification by proportional modifiers, as in partially/fully known opinions. Interestingly, although known behaves like a closed-scale adjective in English, it behaves like an open scale adjective e.g. French, Spanish, and Catalan insofar as it accepts modification by the equivalent of very in these languages (e.g. Catalan molt conegut, 'very known'). See also note 10 , below.

10. Understood, heard, and certain other similar predicates could be argued to correspond to stative rather than nonstative verbs. However, even on their stative reading, the objects of the corresponding verbs manifest a characteristic similar to Mapping to Objects. It is possible for one to stand in these relations to not just the referent of the object as a whole, but also to proper parts of it, and we routinely measure e.g. the depth of or progress in our understanding in terms of the quantity or depth of the facts we understand. The same is true of known, and thus could explain its exceptional behavior.

\section{References}

Bartsch, R. and T. Vennemann. (1973) Semantic Structures: A Study in the Relation between Syntax and Semantics, Athäenum Verlag, Frankfurt.

Bierwisch, M. (1989) "The Semantics of Gradation”, in M. Bierwisch and E. Lang (eds.), Dimensional Adjectives, Springer-Verlag, Berlin.

Bolinger, D. (1972) Degree Words, Mouton, The Hague.

Borer, H. (1998) "Deriving Passive without Theta Roles", in S.G. Lapointe et al. (eds.), Morphological Interfaces, CSLI Publications, Stanford.

Dowty, D.R. (1991) “Thematic Proto-Roles and Argument Selection”, Language 67, 547-619. 
Hay, J. (1998) “The Non-Uniformity of Degree Achievements", paper presented at the 72nd Annual Meeting of the Linguistics Society of America, New York.

Hay, J., C. Kennedy, and B. Levin. "Scale Structure Underlies Telicity in Degree Achievements", this volume.

Hellan, L. (1981) Towards an Integrated Analysis of Comparatives, Narr, Tübingen. Jackendoff, R. (1996) "The Propert Treatment of Measuring Out, Telicity, and Perhaps Even Quantification in English", Natural Language and Linguistic Theory 14, 305-354.

Kamp, J.A.W. (1975) "Two Theories of Adjectives", in E. Keenan (ed.), Formal Semantics of Natural Language, Cambridge University Press, Cambridge.

Kennedy, C. (1999) Projecting the Adjective: The Syntax and Semantics of Gradability and Comparison, Garland, New York.

Klein, E. (1991) "Comparatives", in A. von Stechow and D. Wunderlich (eds.), Semantik: Ein Internationales Handbuch der Zeitgenössischen Forschung, Walter de Gruyter, Berlin.

Klein, E. (1980) “A Semantics for Positive and Comparative Adjectives”, Linguistics and Philosophy 4, 1-45.

Krifka, M. (1992) "Thematic Relations as Links between Nominal Reference and Temporal Constitution", in I. Sag and A. Szabolcsi (eds.), Lexical Matters, CSLI Publications, Stanford, CA. 29-53

Krifka, M. (1989) "Nominal Reference, Temporal Constitution and Quantification in Event Semantics”, in R. Bartsch, J. van Benthem, and P. van Emde Boas (eds.), Semantics and Contextual Expression, Foris, Dordrecht.

Lehrer, A. (1985) "Markedness and Antonymy”, J. of Linguistics 21, 397-429.

Levin, B. and M. Rappaport. (1986) "The Formation of Adjectival Passives", Linguistic Inquiry 17, 623-661.

Ludlow, P. (1989) "Implicit Comparison Classes”, Linguistics and Philosophy 12, $519-533$.

McConnell-Ginet, S. (1973) Comparative Constructions in English: A Syntactic and Semantic Analysis, Ph.D. dissertation, University of Rochester.

Ramchand, G.C. (1997) Aspect and Predication, Clarendon Press, Oxford.

Sapir, E. (1944) “Grading: A Study in Semantics”, Phil. of Science 11, 93-1 16.

Seuren, P.A.M. (1978) "The Structure and Selection of Positive and Negative Gradable Adjectives," Papers from the Parasession on the Lexicon, Chicago Linguistic Society, Chicago, IL, 336-346.

von Stechow, A. (1984a) "Comparing Semantic Theories of Comparison”, Journal of Semantics 3, 1-77.

von Stechow, A. (1984b) “My Reply to Cresswell's, Hellan's, Hoeksema's and Seuren's comments”, Journal of Semantics 3, 183-199.

Tenny, C.L. (1995) "How Motion Verbs are Special: The Interaction of Semantic and Pragmatic Information in Aspectual Verb Meanings," Pragmatics and Cognition 3, 31-73.

: Yumoto, Y. (1991) "The Role of Aspectual Features in Morphology”, English Linguistics 8, 104-123. 